\title{
MODELLING DAMAGE GROWTH AND FAILURE IN ELASTIC MATERIALS WITH RANDOM DEFECT DISTRIBUTIONS
}

\author{
By A.B. Lennon and P.J. Prendergast* \\ Centre for Bioengineering, Department of Mechanical Engineering, Trinity College, \\ Dublin
}

[Received 10 March 2003. Read 19 June 2003. Published 17 December 2004.]

\begin{abstract}
Failure in materials under cyclic loading occurs by the growth and propagation of cracks. If they are sufficiently numerous, the cracks can be considered to be continuously distributed in the material. In this paper, a mathematical model is proposed to predict failure in materials with defects in the form of randomly distributed pores. The model includes anisotropic damage accumulation, the effects of crack closure and the coupling of elasticity with damage. Defects are modelled as pre-existing isotropic damage. A numerical implementation of the model is used to predict failure in specimens with both high and low defect densities, under a cyclical load. Comparison of the predicted failure time with the experimental values shows that the approach captures the variability found in the failure of the specimens. Therefore the model provides a computational scheme that can be used to predict the variability in the failure of load-bearing structures operating under cyclical loads.
\end{abstract}

\section{Introduction}

Prediction of structural failure is one of the central problems in the mechanics of solid materials. The first work on failure prediction for materials that undergo plastic deformation before failure was reported by Huber and others at the turn of the nineteenth century [27], but it was not until Wöhler [29] that a model was proposed for how materials would fail in response to cyclically varying loads. He reported diagrams of stress (S) versus number of cycles to failure $(\mathrm{N})$, and now $\mathrm{S}-\mathrm{N}$ (Wöhler) curves are the most usual approach for predicting failure in engineering applications. However, Wöhler's approach was empirical and did not involve a mathematical model of damage evolution in the material. In a component subjected to a cyclical load, cracks grow from various internal flaws until one crack becomes dominant. This dominant crack becomes critical in the sense that, when it reaches a particular length, it becomes unstable and propagates rapidly. Griffith [8] proposed that the stress intensity in front of a crack could be compared with the fracture toughness to determine whether or not the crack is critical. Later, Paris and Erdogan [21] presented a fatigue crack growth equation relating the rate of crack growth to the stress intensity ahead of a crack tip.

There are many instances, however, where the failure of a component is not governed by the growth of a single crack; rather, many microcracks grow simultaneously and coalesce. In particular, the high strength materials used for

*Corresponding author; e-mail: pprender@tcd.ie 
nuclear power stations are designed to be damage-tolerant and therefore to accumulate damage before failure. In the analysis of these failures, Kachanov [9] introduced the concept of damage continuously distributed throughout the solid and proposed a damage variable as an internal state variable describing the state of degradation of the material. The continuum damage, $D$, may be considered as the local loss of cross-sectional area, giving an effective stress, $\tilde{\sigma}$, as

$$
\tilde{\sigma}=\frac{\sigma}{1-D}
$$

where failure occurs at $D=1$. Lemaitre and Chaboche [13] describe how vector and tensorial representations of damage may be developed and show damage evolution equations for components under cyclical loading.

An issue yet to be fully addressed in problems relating to failure of mechanical engineering components under cyclical loading is that real failures occur with significant variation. Even for components where the stress state is well controlled (such as samples used for materials testing) the stress vs cycles-to-failure data is usually very scattered. This is because the process of damage accumulation is stochastic in nature. In this paper we use continuum damage mechanics to develop a computational methodology for prediction of degradation of materials under cyclical loading. The approach is tested against experimental data obtained for fatigue tests of polymethylmethacrylate (Perspex) [18], which has been shown to be prone to fatigue damage accumulation from pores $[15 ; 19]$.

\section{Methods}

\subsection{Coupling of elastic properties with damage}

An assumption of elastic strain energy equivalence [7; 25] was used to account for loss of material stiffness in planes with damage. This approach defines an effective stress tensor, $\tilde{\boldsymbol{\sigma}}$, and effective strain tensor $\tilde{\boldsymbol{\epsilon}}$, such that the strain energy of the damaged material is equivalent to the strain energy of the undamaged material:

$$
\frac{1}{2} \boldsymbol{\sigma}: \boldsymbol{\epsilon}=\frac{1}{2} \tilde{\boldsymbol{\sigma}}: \tilde{\boldsymbol{\epsilon}}
$$

where

$$
\boldsymbol{\sigma}=\tilde{\boldsymbol{E}}: \boldsymbol{\epsilon} \quad \text { and } \quad \tilde{\boldsymbol{\sigma}}=\boldsymbol{E}: \tilde{\boldsymbol{\epsilon}},
$$

and $\boldsymbol{\sigma}, \boldsymbol{\epsilon}, \boldsymbol{E}$, and $\tilde{\boldsymbol{E}}$ are the Cauchy stress tensor, small strain tensor, elastic stiffness tensor and effective elastic stiffness tensor, respectively. If a fourth-order damage effect tensor, $\boldsymbol{M}$, is assumed to transfer the stress tensor to its effective counterpart as

$$
\tilde{\boldsymbol{\sigma}}=\boldsymbol{M}: \boldsymbol{\sigma},
$$

then suitable manipulation yields [25]

$$
\tilde{\boldsymbol{E}}=\boldsymbol{M}^{-1}: \boldsymbol{E}: \boldsymbol{M}^{-T} \text {. }
$$


In general, $\tilde{\boldsymbol{\sigma}}$ need not be symmetric according to (2.3) - to avoid the added complexity of polar media, $\boldsymbol{M}$ can be chosen so as to maintain symmetry for the effective stress. Several symmetry preserving transformations have been proposed based on the values of a second-rank damage tensor, $\boldsymbol{d}$ [28]. This allows a relatively simple and physically intuitive method of representing anisotropic damage. In the current model the damage effect tensor is assumed to take the following form [28]:

$$
M_{i j k l}=\left(\delta_{i k}-d_{i k}\right)^{-1 / 2}\left(\delta_{j l}-d_{j l}\right)^{-1 / 2},
$$

where $\delta_{i j}$ is the Kronecker delta. This expression takes its simplest form in a coordinate system aligned with the principal damage directions. However, the anisotropy induced by damage requires that equation (2.4) most often be evaluated in a reference coordinate system. Assuming the undamaged material is isotropic, the matrix form of (2.4) can be expressed in the principal damage coordinate system as

$$
[\tilde{\boldsymbol{E}}]=\left(\begin{array}{cc}
Q_{1} & 0 \\
0 & Q_{2}
\end{array}\right)
$$

where

$$
\begin{aligned}
& {\left[Q_{1}\right]=\left(\begin{array}{ccc}
\left(1-d_{11}\right)^{2} E_{11} & \left(1-d_{11}\right)\left(1-d_{22}\right) E_{12} & \left(1-d_{11}\right)\left(1-d_{33}\right) E_{12} \\
\left(1-d_{11}\right)\left(1-d_{22}\right) E_{12} & \left(1-d_{22}\right)^{2} E_{11} & \left(1-d_{22}\right)\left(1-d_{33}\right) E_{12} \\
\left(1-d_{11}\right)\left(1-d_{33}\right) E_{12} & \left(1-d_{22}\right)\left(1-d_{33}\right) E_{12} & \left(1-d_{33}\right)^{2} E_{11}
\end{array}\right)} \\
& {\left[Q_{2}\right]=\left(\begin{array}{ccc}
\left(1-d_{11}\right)\left(1-d_{23}\right) E_{44} & 0 & 0 \\
0 & \left(1-d_{22}\right)\left(1-d_{33}\right) E_{44} & 0 \\
0 & 0 & \left(1-d_{33}\right)\left(1-d_{11}\right) E_{44}
\end{array}\right)}
\end{aligned}
$$

and

$$
E_{11}=\frac{\mathrm{E}(1-v)}{(1+v)(1-2 v)}, \quad E_{12}=\frac{\mathrm{E} v}{(1+v)(1-2 v)}, \quad E_{44}=\frac{\mathrm{E}}{2(1+v)},
$$

where E and $v$ represent Young's modulus and Poisson's ratio, respectively.

For planar microcracks, it is possible that cracks may close under compressive loading. This should cause the material to regain stiffness normal to the cracked plane. Several approaches have been proposed to account for this effect, e.g. [12; 16; 24]. However, these models have been shown to exhibit either discontinuities in stress response on closure or loss of symmetry in the stiffness tensor [4]. A spectral decomposition of the stiffness tensor using the principal planes of the damage tensor has been shown to avoid these problems [5] and has formed the approach taken in this study. Firstly, a fourth-order projection tensor, $\boldsymbol{P}^{d_{i}}$ (superscript $d_{i}$ implies the $i$ th principal damage component), can be constructed from the $i$ th normalised principal damage vector, $\hat{\boldsymbol{e}}^{d_{i}}$, as

$$
\boldsymbol{P}^{d_{i}}=\hat{\boldsymbol{e}}^{d_{i}} \otimes \hat{\boldsymbol{e}}^{d_{i}} \otimes \hat{\boldsymbol{e}}^{d_{i}} \otimes \hat{\boldsymbol{e}}^{d_{i}} .
$$

The strain normal to this plane, $\epsilon^{d_{i}}$, can be decomposed from the total strain tensor according to 


$$
\boldsymbol{\epsilon}^{d_{i}}=\boldsymbol{P}^{d_{i}}: \boldsymbol{\epsilon}
$$

In writing the matrix form of (2.8) the strain tensor is written in the form of engineering strains, i.e. $\{\gamma\}^{T}=\left\{\epsilon_{11}, \epsilon_{22}, \epsilon_{33}, 2 \epsilon_{12}, 2 \epsilon_{23}, 2 \epsilon_{31}\right\}$, which gives the following form for the projection matrix:

$$
\left\{\boldsymbol{\gamma}^{d_{i}}\right\}=\left[\boldsymbol{P}^{d_{i}}\right]\{\gamma\},
$$

where $\left\{\gamma^{d_{i}}\right\}$ is the projection of engineering strain for the $i$ th principal damage direction and

$$
\left[\boldsymbol{P}^{d_{i}}\right]=\left(\begin{array}{cccccc}
a^{4} & a^{2} b^{2} & a^{2} c^{2} & a^{3} b & a^{2} b c & a^{3} c \\
a^{2} b^{2} & b^{4} & b^{2} c^{2} & a b^{3} & b^{3} c & a b^{2} c \\
a^{2} c^{2} & b^{2} c^{2} & c^{4} & a b c^{2} & b c^{3} & a c^{3} \\
2 a^{3} b & 2 a b^{3} & 2 a b c^{2} & 2 a^{2} b^{2} & 2 a b^{2} c & 2 a^{2} b c \\
2 a^{2} b c & 2 b^{3} c & 2 b c^{3} & 2 a b^{2} c & 2 b^{2} c^{2} & 2 a b c^{2} \\
2 a^{3} c & 2 a b^{2} c & 2 a c^{3} & 2 a^{2} b c & 2 a b c^{2} & 2 a^{2} c^{2}
\end{array}\right),
$$

where $\{a, b, c\}$ are the coefficients of $\hat{\boldsymbol{e}}^{d_{i}}$.

The model proposed in [5] takes the form

$$
\tilde{\boldsymbol{E}}=\tilde{\boldsymbol{E}}+\sum_{i=1}^{3} \mathrm{H}\left(-\operatorname{Tr}\left(\boldsymbol{P}^{d_{i}}: \boldsymbol{\epsilon}\right)\right) \boldsymbol{P}^{d_{i}}:(\boldsymbol{E}-\tilde{\boldsymbol{E}}): \boldsymbol{P}^{d_{i}},
$$

where $\operatorname{Tr}()$ is the trace operator and $\mathrm{H}()$ is the Heaviside function (equal to zero for positive normal strain and one for negative strain). The effect of this transformation can be understood by consideration of the simplest case of a principal damage direction that is coaxial with one of the global reference axes. For example, consider the case $\hat{\boldsymbol{e}}^{d_{1}}=\{1,0,0\}$. Evaluation of (2.10) leaves only the $a^{4}$ term, and substitution into (2.9) gives

$$
\left\{\gamma^{d_{1}}\right\}^{T}=\left\{\epsilon_{11}, 0,0,0,0,0\right\} .
$$

Thus, it can be seen that the trace of the above projected strain is the strain normal to a crack. Substituting $d_{11}=1$ into (2.6) gives the matrix form of

$$
[\boldsymbol{E}]-[\tilde{\boldsymbol{E}}]=\left(\begin{array}{cccccc}
E_{11} & E_{12} & E_{12} & 0 & 0 & 0 \\
E_{12} & 0 & 0 & 0 & 0 & 0 \\
E_{12} & 0 & 0 & 0 & 0 & 0 \\
0 & 0 & 0 & E_{44} & 0 & 0 \\
0 & 0 & 0 & 0 & 0 & 0 \\
0 & 0 & 0 & 0 & 0 & E_{44}
\end{array}\right) .
$$

The matrix form of the projection contained in (2.11) is thus

$$
\left[\boldsymbol{P}^{d_{1}}\right]^{T}([\boldsymbol{E}]-[\tilde{\boldsymbol{E}}])\left[\boldsymbol{P}^{d_{1}}\right]=\left(\begin{array}{cccccc}
E_{11} & 0 & 0 & 0 & 0 & 0 \\
0 & 0 & 0 & 0 & 0 & 0 \\
0 & 0 & 0 & 0 & 0 & 0 \\
0 & 0 & 0 & 0 & 0 & 0 \\
0 & 0 & 0 & 0 & 0 & 0 \\
0 & 0 & 0 & 0 & 0 & 0
\end{array}\right) .
$$


This simple case illustrates that the effect of the projection operation is to restore only the stiffness normal to the crack plane when closure occurs.

\subsection{Damage growth}

Many materials have been observed to sustain microcrack damage perpendicular to tensile principal stress - this behaviour will be the subject of the current model. Dependence on tensile principal stresses requires the use of a coordinate system defined by the principal stress directions. After rotation of the damage tensor to a coordinate system aligned with the principal stress vectors, the damage growth rate in the plane normal to each principal stress direction conformed to an empirically derived damage evolution equation,

$$
\frac{\partial d_{i i}}{\partial n}=\alpha_{i} \frac{n^{\alpha_{i}-1}}{N_{F_{i}}^{\alpha_{i}}} .
$$

where $N_{F_{i}}$ is the number of cycles required to cause failure at the applied maximum principal stress, $\sigma_{i i}^{M}, n$ is the number of applied loading cycles and $\alpha_{i}=f\left(\sigma_{i i}^{M}\right)$ is a stress dependent exponent [17]. Nonlinear accumulation results from the stress dependence of the exponent for arbitrary loading sequences (e.g. high stress followed by low stress) [3]; this requires special treatment when adding damages for arbitrary load sequences. Each damage component normal to a tensile principal stress was calculated from

$$
d_{i i}=\left(\frac{n_{i}^{e q}+\Delta n}{N_{F_{i}}}\right)^{\alpha_{i}},
$$

where $n_{i}^{e q}$ is the number of cycles required to achieve an equivalent damage on the damage curve for the current stress (see Fig. 1) and $\Delta n$ is the applied cycle increment. The definition of $\alpha(\sigma)$ used here is:

$$
\begin{gathered}
\alpha(\sigma)=\frac{\sigma-\beta}{\gamma} ; \quad \sigma>\beta+\gamma, \text { and } \\
\alpha(\sigma)=1 ; \quad 0<\sigma \leq \beta+\gamma .
\end{gathered}
$$

Note that the second of these expressions prevents the unphysical phenomena of very rapid damage growth at low stresses (see Fig. 2) and instantaneous rupture at $\alpha=\beta$ (i.e. if only (2.17a) were used).

For the general case of continuously changing principal stress directions, the rotation of principal stresses must be taken into account when applying the damage growth equation. Skrzypek [25] proposed the following form for an objective damage rate tensor, $\boldsymbol{\nabla}$ :

$$
\stackrel{\nabla}{\boldsymbol{d}}=\frac{\partial \boldsymbol{d}}{\partial n}-\boldsymbol{d}^{T} \cdot \boldsymbol{S}-\boldsymbol{S}^{T} \cdot \boldsymbol{d},
$$

where $\frac{\partial \boldsymbol{d}}{\partial n}$ is the damage rate tensor of (2.15) and $\boldsymbol{S}$ is a skew symmetric spin tensor due to rotation of principal stress directions. 


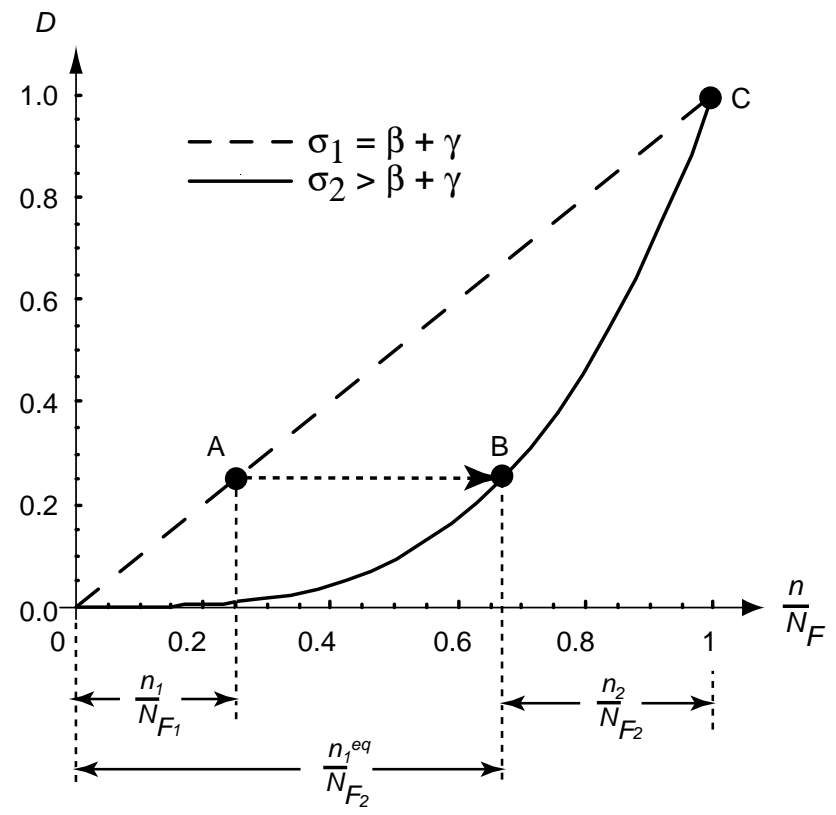

FIG. 1-Definition of equivalent number of cycles used in equation (2.16), i.e. moving from point $\mathrm{A}$ on the initial curve to a point of equivalent damage, $\mathrm{B}$, on the second curve and defining the equivalent elapsed number of cycles to cause this damage. Point $\mathrm{C}$ corresponds to failure of the specimen. This also illustrates the nonlinear load-sequence effect of the damage rule, i.e. life fractions do not sum to unity, as would be the case for the well known Palmgren-Miner cumulative damage rule.

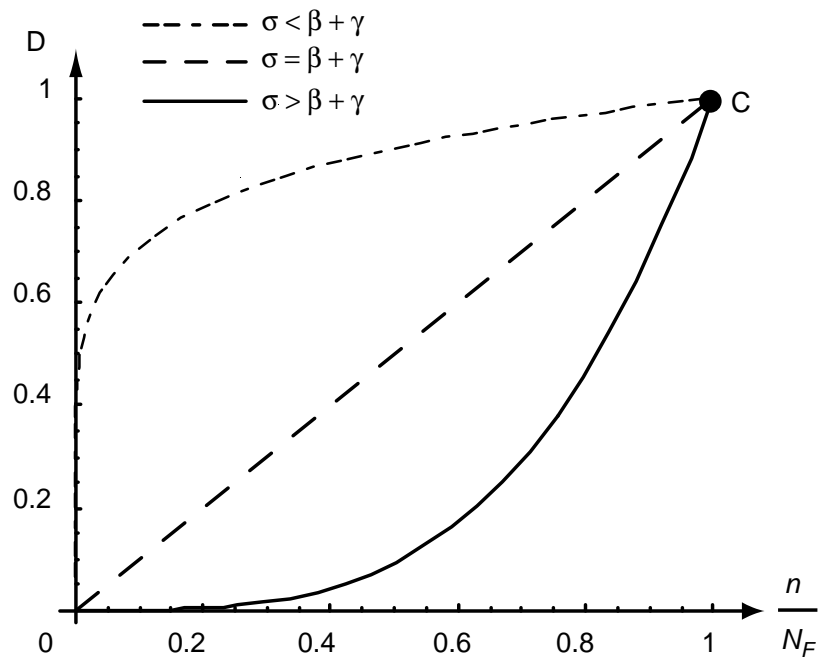

FIG. 2 - Nonlinear damage curves for three stress ranges. Note the very rapid rise in damage for $\sigma<\beta+\gamma$. Furthermore, substitution of $\sigma=\beta$ into $(2.17 a)$ would result in an instantaneous rupture, i.e. $d_{i i}=1$. 


\subsection{Porosity}

The material reported in the experiments of Murphy and Prendergast [18; 19] fails from cracks originating from pores formed on polymerisation. This type of damage accumulation has also been observed when the material forms a layer in a more complex structure made of several other materials [15]. These random defects were introduced into the model, and the interaction between porosity and damage accumulation was achieved through coupling porosity with elastic properties. Pores were modelled as isotropic damage tensors with the capability of crack closure removed. Equation (2.5) was then used to calculate a pore effect tensor, $\boldsymbol{M}_{\mathrm{p}}$, replacing $d_{i i}$ with $p_{i i}$, and (2.4) was used to calculate the stiffness loss due to porosity. The effective stress was calculated by first evaluating the effective strain and then substituting into (2.2), i.e.

$$
\tilde{\boldsymbol{\epsilon}}=\boldsymbol{M}_{\mathrm{p}}^{-T}: \boldsymbol{\epsilon} \text { and } \tilde{\boldsymbol{\sigma}}=\boldsymbol{E}: \tilde{\boldsymbol{\epsilon}}
$$

The principal values of this stress tensor were then used in the calculation of damage accumulation according to equations (2.16) and (2.17).

\subsection{Numerical solution}

A finite element code was written to carry out the simulations of damage accumulation [14]. The cycle increment was estimated by finding the next integration point in the finite element mesh that would reach a critical principal damage value. Damage was assumed to remain uncoupled from elastic properties in a given direction until complete failure was predicted for that direction, at which time coupling was introduced according to Section 2.1. First, damage had to be rotated from a global reference coordinate system to a coordinate system defined by the principal stress axes. An equivalent number of elapsed cycles was then calculated for each principal stress direction, and the damage growth equation (2.16) was used with a trial cycle increment, $\Delta n$. To account for possible rotation of the damage tensor, the principal damage directions were checked for every trial cycle increment to see if any additional cracks had been initiated. A bisection algorithm was then applied to find the cycle increment required to cause at least one new crack in the integration point. The minimum cycle increment for the mesh was then chosen as the global cycle increment; damage accumulation of all points was then calculated.

A random number generator was used to generate a distribution of pores within the material. Two parameters were used to control the type of porosity generated: mean specimen porosity ( $\%$ volume) and pore radii. First, a distribution was generated to determine whether a particular integration point contained a pore by specifying a tolerance about the mean value of the distribution - points with values inside the tolerance were deemed to contain a pore. A second distribution was used to assign pore radii to the points, and these values were used to calculate individual pore volumes. To decrease the dependence on a particular mesh discretisation, pores with volumes larger than the volume attributable to a given integration point of the finite element model were allowed to populate regions occupied by neighbouring integration points. On completing a pass through the mesh, the total 
specimen porosity was checked. If the value lay within two standard deviations of the desired mean, the pore distribution was accepted; otherwise the tolerance was adapted to accept either more or less pores and iteration continued until an acceptable distribution was achieved. Porosity tensors were then generated for integration points by setting the normal (diagonal) components equal to the volumetric fraction of a pore for each point:

$$
p_{i i}=\frac{v_{p}}{v_{i p}}
$$

where $v_{p}$ is the pore volume and $v_{i p}$ is the integration point volume; other components were set to zero.

\subsection{Application of the modelling scheme to fatigue of an acrylic polymer}

Acrylic bone cement is a self-curing acrylic resin, chemically similar to Perspex (i.e. consists primarily of polymethylmethacrylate), and is prone to developing widely differing porosity distributions depending on preparation technique. Failure data for two preparation techniques were available [18]: (i) high-porosity, due to entrapment of air during mixing, and (ii) low-porosity, due to evacuation of air during mixing. The relationship between stress and number of cycles to failure for the pore-free cement was

$$
\sigma=-6.04 \log _{10} N_{F}+53.46
$$

A Young's modulus of $2.8 \mathrm{GPa}$ and Poisson's ratio of 0.33 were used. A uniform tension was applied to one face of the specimen to give the required nominal stress in the central section of a pore-free specimen. Nodal displacements on the opposite face were set to zero in the direction of the load; nodes defining the central axes of the restrained face were also fixed in the other two orthogonal directions to prevent rigid body motions.

To replicate the experiments carried out in the laboratory, eight simulations for each of four stress levels (25MPa, 21 MPa, 17.5MPa, and 13MPa) were performed for both high defect density and low defect density cement. Each of the 64 simulations had a different pore distribution generated by the methods described in Section 2.4.

The parameters used to generate the pore distributions were obtained by fitting the mean and standard deviation of total volume fraction of porosity as well as mean and standard deviation of pore radius at one stress level $(21 \mathrm{MPa})$. When similar maximum and minimum failure lives were predicted from a set of eight simulations the parameters were applied to the rest of the stress levels. One set of specimens (high-porosity) were characterised by a large number of small pores, whereas the other set of specimens (low-porosity) were characterised by a few pores of relatively large radius (Fig. 3). The values used to achieve these distributions, and the resulting average total specimen volume fraction of pores, are shown in Tables 1 and 2 .

Simulations of damage accumulation were carried out for all specimens for each of the characteristic defect distributions shown in Fig. 3. 

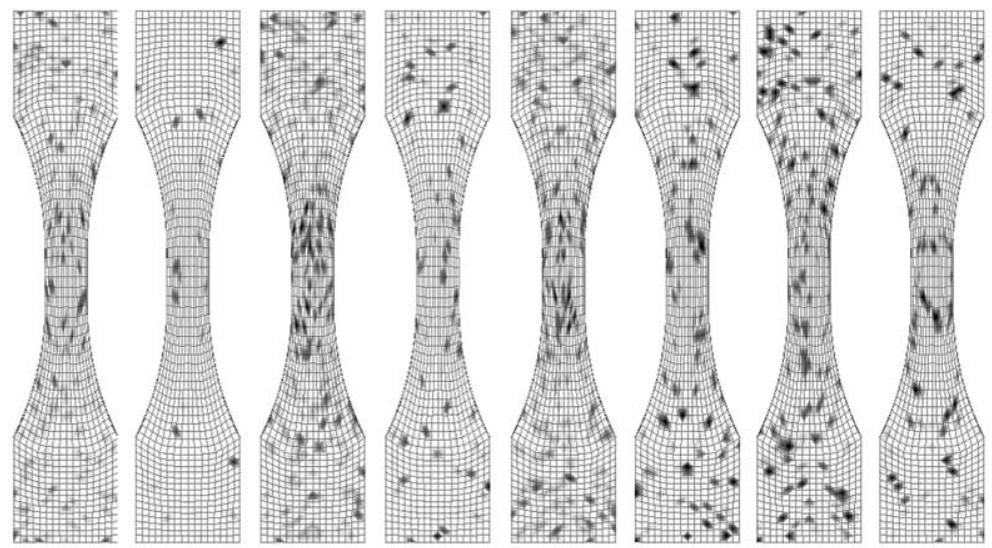

(a) High defect density specimens
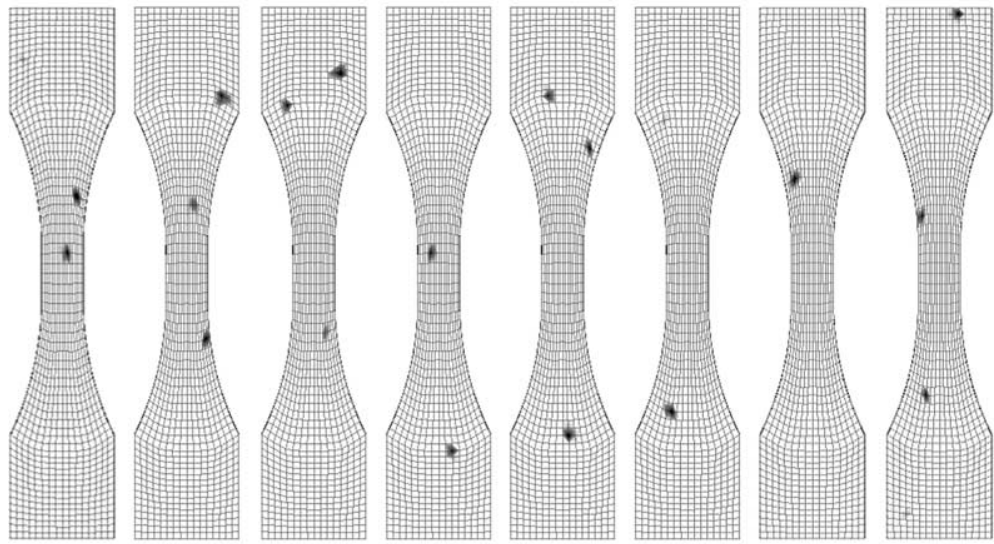

(b) Low defect density specimens

FIG. 3-Simulated porosity distributions of (a) high-porosity specimens and (b) low-porosity specimens for the $13 \mathrm{MPa}$ stress level. A greyscale from 0 to 1 is used to indicate pore volume fraction averaged at nodes.

\section{Results}

For high-porosity specimens, damage always initiated from pores, sometimes from several sites simultaneously (Fig. 4a). However, low-porosity specimens sometimes failed from the sites of nominal peak stress, predicted to be the point of tangency

TABLE 1 - Mean and standard deviation (SD) of percentage porosity and pore radius used as input for high and low defect densities.

\begin{tabular}{lcclcc}
\hline & \multicolumn{2}{c}{ High porosity } & & \multicolumn{2}{c}{ Low porosity } \\
\cline { 2 - 3 } \cline { 5 - 6 } & Mean & $S D$ & & Mean & $S D$ \\
\hline Porosity (\% vol) & 5.00 & $(2.00)$ & & 0.25 & $(0.125)$ \\
Radius (mm) & 0.20 & $(0.60)$ & & 1.75 & $(0.05)$ \\
\hline
\end{tabular}


TABLE 2 - Mean and standard deviation (SD) of total specimen volume fraction of pores for each mixing method achieved in the simulations. No radius data is presented as only pore volume fractions were stored in the simulations.

\begin{tabular}{lcclccc}
\hline & \multicolumn{2}{c}{ High porosity } & & \multicolumn{2}{c}{ Low porosity } \\
\cline { 2 - 3 } \cline { 5 - 6 } Mean & $S D$ & & Mean & $S D$ \\
\hline Porosity $(\% \mathrm{vol})$ & 5.76 & $(2.02)$ & & 0.34 & $(0.10)$ \\
\hline
\end{tabular}

between the straight central section and the curved sections; these can be seen as the symmetric damage patterns (end-point damage patterns shown in Fig. 4b). In general, failure occurred either within or near the gauge for both high-porosity and low-porosity specimens.

Damage evolution curves show that, for higher stresses, damage accumulated more nonlinearly for high defect density and low defect density specimens (Fig. 5).

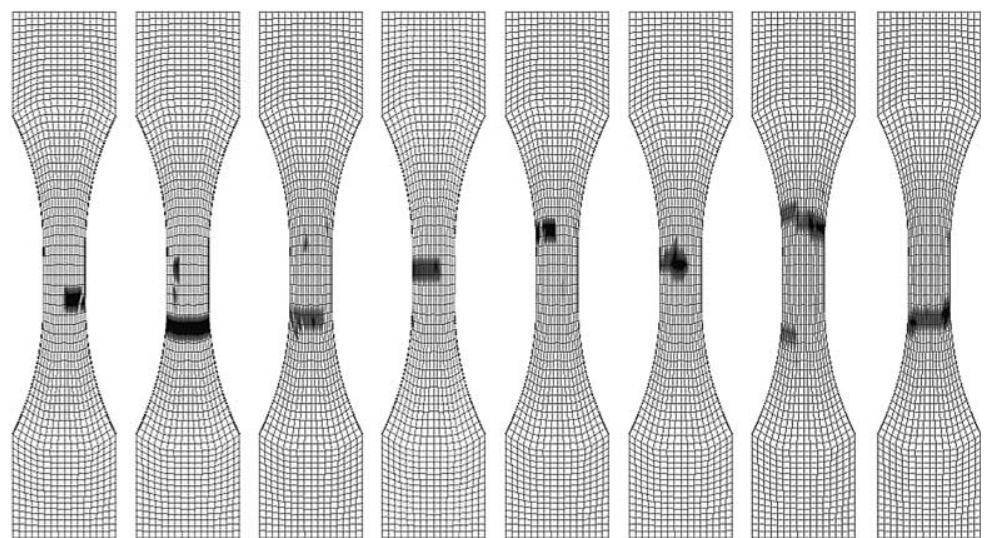

(a) 'End point' damage patterns in high defect density specimens

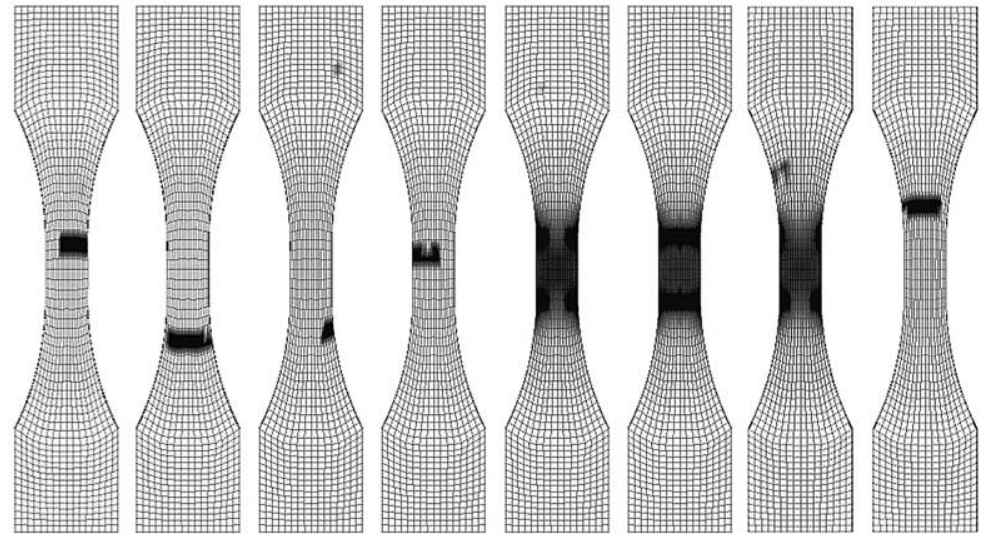

(b) 'End point' damage patterns in low defect density specimens

FIG. 4-Simulated damage distributions of (a) high-porosity specimens and (b) low-porosity specimens for the 13MPa stress level. A greyscale from 0 to 1 is used to indicate damage averaged at nodes. 


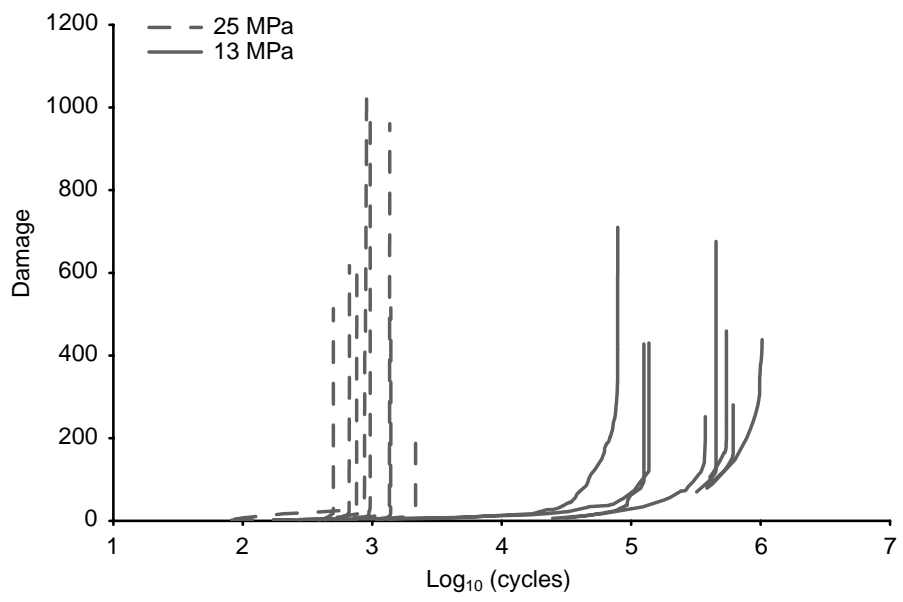

(a) High defect density specimens

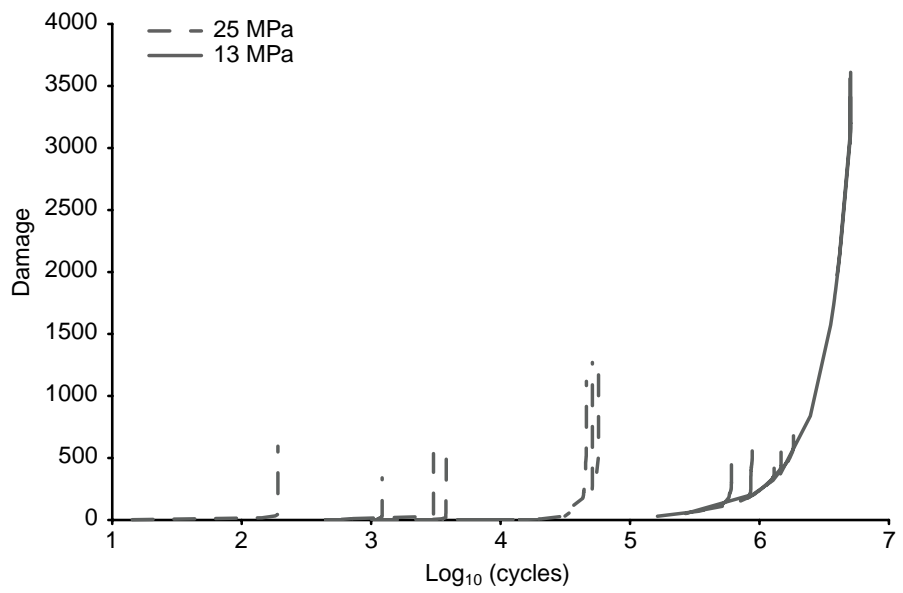

(b) Low defect density specimens

FIG. 5-Simulated damage evolution for (a) high-porosity specimens and (b) low-porosity specimens for $25 \mathrm{MPa}$ and $13 \mathrm{MPa}$ stress levels. Damage was calculated as the trace of the damage tensor for each integration point and summed over all integration points. The larger scale for the low-porosity data set is due to the three specimens with symmetric damage patterns (Fig. $4 \mathrm{~b}$ ).

A much greater magnitude of damage accumulation before failure is apparent for the samples at the low stress level in the low-porosity data. This can be explained by the symmetric damage patterns of these specimens (Fig. 4b), as well as the more uniform stress that occurs in the gauge of the specimen. Because of the relatively uniform stress in the gauge, damage accumulation is also more uniform, while for the case of a critical pore outside the gauge, the stress raising effect and consequent damage accumulation are more localised.

The modelling approach captured the variable failure life behaviour of polymethylmethacrylate. Least squares regression lines were used to compare 
the overall fit of both experimental and numerical results (Fig. 6). Quite similar stress vs cycles-to-failure curves to those found experimentally were predicted for both high-porosity and low-porosity sets (see Fig. 6). The regression equations for the high defect density specimens are:

$$
\begin{array}{ll}
\text { Experiment } & \sigma=-3.76 \log _{10} N_{F}+34.95 \\
\text { Simulation } & \sigma=-3.58 \log _{10} N_{F}+33.19
\end{array}
$$

and for the low defect density specimens are:
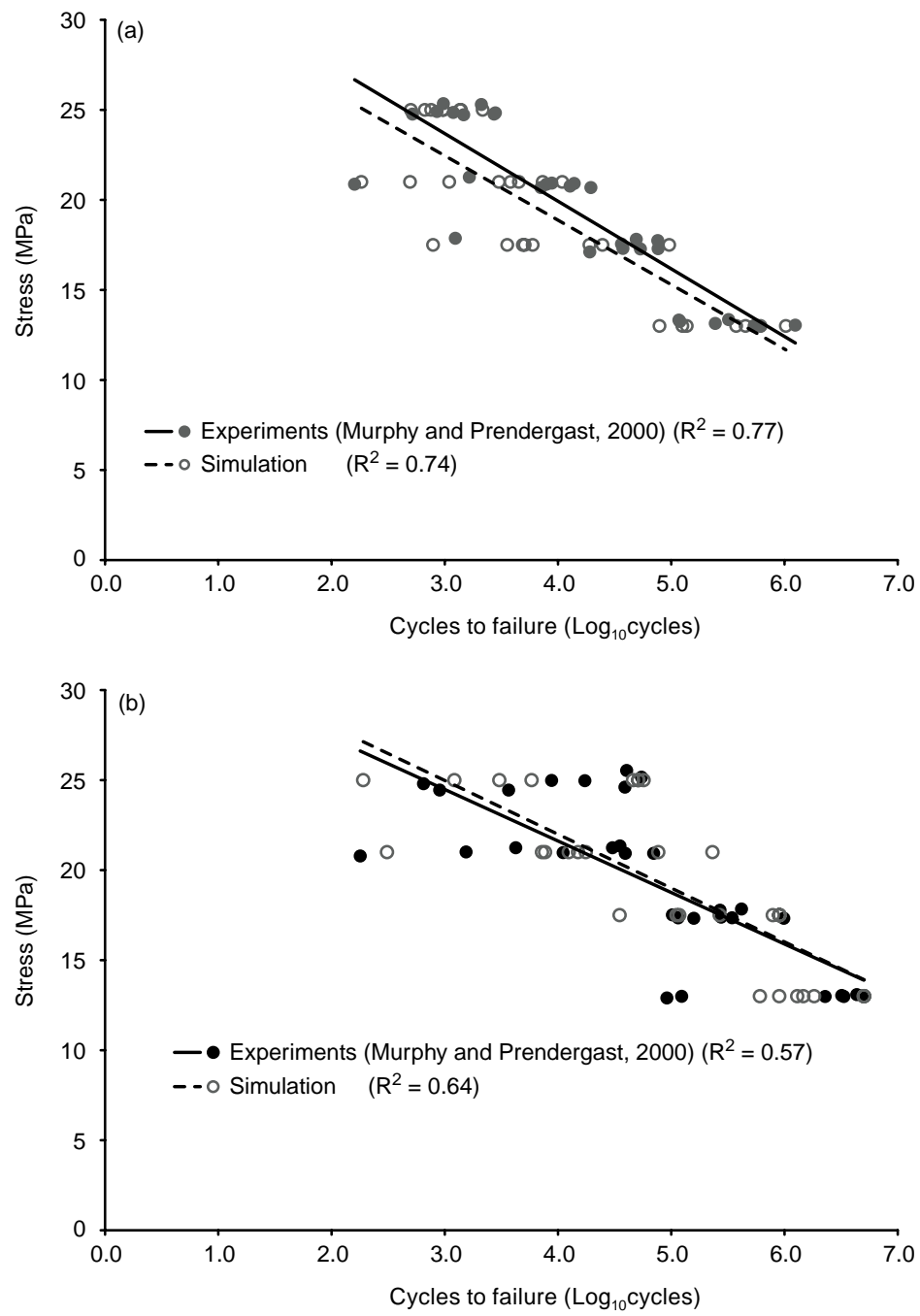

FIG. 6-Comparison of (a) high-porosity and (b) low-porosity regression lines of [18] with regression lines fitted to data of simulated tests. 


$$
\begin{array}{cc}
\text { Experiment } & \sigma=-2.86 \log _{10} N_{F}+33.06 \\
\text { Simulation } & \sigma=-2.99 \log _{10} N_{F}+33.97
\end{array}
$$

where $\sigma$ is the applied stress and $N_{F}$ is the number of cycles to failure.

Significance tests were used to determine whether or not the simulated data could be statistically discriminated from the experimental data. For such a test, significance values of $p>0.05$ would indicate that the results from experiment and simulations cannot be distinguished and higher $p$ values indicate a greater probability that the specimens are similarly distributed. Direct comparison of average failure life at each stress level for the simulated data set with the corresponding experimental data set shows that the experimental and simulated results are likely to form part of the same distribution (Table 3).

Finally, plotting regression lines through the means of the experimental data at each stress level shows a clear increase in average fatigue life for the low-porosity specimens - a similar increase was predicted by the simulations (Fig. 7).

\section{Discussion}

A modelling scheme was developed to predict the variability of failure of materials with random defect distributions. However, several limitations apply to it:

(1) Damage was defined as a local internal state variable and therefore the effect of crack interactions at high damage concentrations is neglected. The lack of nonlocality in the definition of damage can also result in localisation instability and mesh dependent solutions [2; 22]. It has been shown that the ill effects of local damage definitions can be avoided if localisation limiters, such as a weighted volume averaging of damage, e.g. [23], are incorporated. Relatively coarse discretisation in comparison to the observed microstructural damage, as used in this study, can also inhibit localisation instability and mesh dependency [1].

TABLE 3-Mean and standard deviation (SD) of failure life for each data set and significance $(p)$ values for

\begin{tabular}{|c|c|c|c|c|c|}
\hline \multirow[t]{2}{*}{ Stress (MPa) } & \multicolumn{2}{|c|}{ Experimental } & \multicolumn{3}{|c|}{ Simulated } \\
\hline & Mean & $S D$ & Mean & $S D$ & $p$ \\
\hline \multicolumn{6}{|l|}{ High porosity } \\
\hline 13 & 462,054 & $(398,793)$ & 419,686 & $(318,887)$ & 0.82 \\
\hline 17.5 & 43,683 & $(26,058)$ & 20,006 & $(31,839)$ & 0.12 \\
\hline 21 & 8,985 & $(6,398)$ & 3,918 & $(3,690)$ & 0.07 \\
\hline 25 & 1,580 & (868) & 1,097 & (532) & 0.20 \\
\hline \multicolumn{6}{|l|}{ Low porosity } \\
\hline 13 & $2,628,680$ & $(1,928,225)$ & $2,652,430$ & $(2,009,684)$ & 0.98 \\
\hline 17.5 & 333,132 & $(285,597)$ & 503,379 & $(402,804)$ & 0.35 \\
\hline 21 & 23,841 & $(24,282)$ & 46,329 & $(78,481)$ & 0.45 \\
\hline 25 & 20,631 & $(21,053)$ & 26,961 & $(26,290)$ & 0.60 \\
\hline
\end{tabular}
a student's $t$-test. 


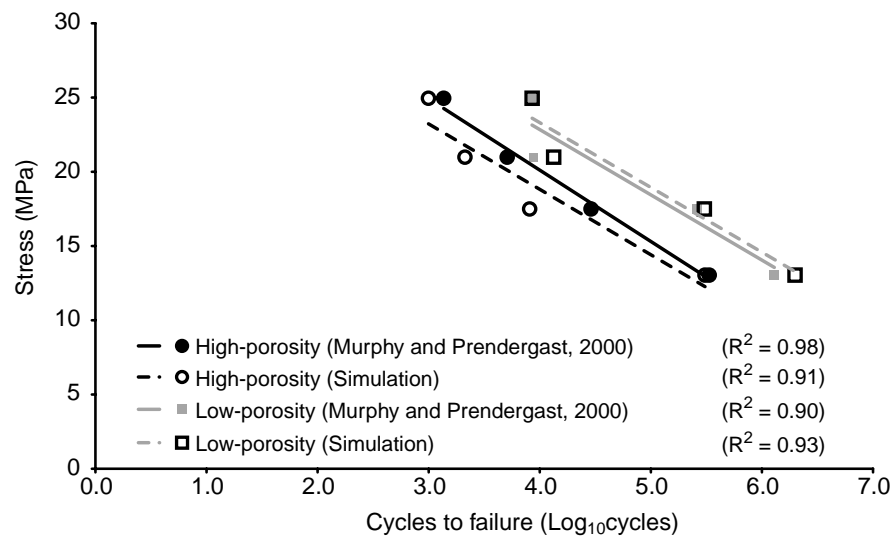

FIG. 7-Average failure life at each stress level and resulting regression lines for high-porosity and lowporosity data of [18] and simulations of present study. The simulated data predicts the average increase in fatigue life for vacuum-mixing found experimentally.

(2) The assumption that damage remained uncoupled from elastic properties until complete failure for a particular plane is justifiable only for materials that show a sudden loss in stiffness near failure, which is the case for polymethylmethacrylate [14] and for fatigue of many other materials. For this type of behaviour, an uncoupled model has been shown to give equivalent results to continuously coupled models with the benefit of reduced computational time [20].

(3) The form of the damage evolution equation considers only maximum tensile stress and thus neglects the possibility that compressive stress could affect damage accumulation (e.g. to resharpen blunted cracks). A possible improvement to the model would be to reformulate the damage evolution equation of Murphy and Prendergast [19] in terms of both stress amplitude and mean stress, such as has been proposed by Chaboche and Lesne [6]. Furthermore, dependence on shear stress is not incorporated in the model of multiaxial damage growth. A more general formulation could incorporate one or more stress invariants. However, a disadvantage of invariant formulations is that they do not always provide a direct prediction of the orientation of the dominant fracture plane.

(4) Anisotropy is limited to orthotropic stiffness reduction, since a second order damage tensor is used to represent the damage state. However, according to Kachanov [10], orthotropic damage is often an acceptable simplification for non-interacting cracks in an isotropic matrix.

(5) Due to the fitting procedure used to obtain the input parameters for the model, the comparison with experimental results cannot strictly be considered as an independent corroboration of the model. However, the fitting procedure was limited to fitting an equivalent range in failure life at one stress level only (i.e. 21MPa). No fit was made to the average failure life at this stress level, and failure at the remaining stress levels was simulated 
without any change in the input parameters. A review of average percentage porosity and mean and maximum pore radius for the two types of defect distribution simulated found that the input parameters were within the range of reported values [14].

In spite of these limitations, several comparisons can be made between the predictions and the experimental data. The computational scheme proved capable of predicting:

(1) similar average lifetimes, and variability in lifetime, as shown by the correspondence between the predicted regression lines and the experimental regression lines (see Fig. 6),

(2) similar ranges of failure life (i.e. maximum and minimum lifetimes, see Fig. 6), and

(3) the average increase in fatigue life for low-porosity specimens over highporosity specimens (see Fig. 7).

An interesting feature of the experimental results was that the high-porosity specimens, although having lower average fatigue life, were statistically more reliable than low-porosity specimens because of their lower variability [18]. This type of behaviour was also predicted by the mathematical model presented here (see Table 3 and Fig. 6). Some explanation for this can be found in the way that defects affect the damage tolerance of a material. The introduction of pores can be considered as creating spatially varying fatigue strength. Krajcinovic [11] has shown that increasing the bandwidth of failure strengths in a material can increase the damage tolerance. Although such materials fail at lower peak loads, they typically undergo much greater and more stable damage accumulation before eventual failure because energy is dissipated continuously from an early stage in a controlled process. In contrast, he showed that materials with relatively uniform strength, i.e. with very few defects, were prone to sudden brittle failure with very little deformation. Failure of such a material may often be governed by a single critical defect; once the failure strength is exceeded at the defect, the initiated crack grows unimpeded. Variation in the size and location of the critical defect between specimens can then lead to more pronounced variability in failure. The low-porosity specimens correspond quite well to this concept so that this may explain the more widely distributed failure life data for the low-porosity specimens in comparison to the more uniformly porous high-porosity specimens found in both the experiments and the numerical simulations.

Finally, incorporation of the proposed modelling approach should be useful in improving existing fatigue damage models for structures containing materials prone to variable defect distributions. For example, a recent model developed by Stolk et al. [26], which included viscoelastic creep in addition to fatigue damage of acrylic bone cement, was applied to the investigation of failure of cemented orthopaedic hip replacements. Their model could be readily adapted to include variable pore distributions using the approach proposed here and this may help in understanding why large variability is seen in clinical failures of such implants. 


\section{Conclusions}

A mathematical model approach was developed to predict failure times of a material that is prone to random defect distributions. The differences in fatigue life behaviour occurring with changes in the characteristics of the defect distributions (i.e. high porosity vs low porosity) were simulated. The results of the simulation were found to be very similar to experimental results. The model is proposed as a method for simulating damage accumulation in components and structures containing materials with defects.

\section{ACKNOWLEDGEMENTS}

The authors acknowledge financial support from the Programme for Research in Third Level Institutions, administered by the Higher Education Authority.

\section{REFERENCES}

[1] Z.P. Bažant and G. Pijaudier-Cabot, Nonlocal continuum damage, localization instability and convergence, Journal of Applied Mechanics 55 (1988), 287-93.

[2] T. Belytschko and D. Lasry, Localization limiters and numerical strategies for strain-softening materials, in J. Mazars and Z.P. Bažant (eds), Cracking and damage — strain localization and size effect, Cachan, France, 1988, France-US Workshop, Elsevier, London, 1989, pp 349-62.

[3] J.L. Chaboche, Continuum damage mechanics: present state and future trends, Nuclear Engineering and Design 105 (1987), 19-33.

[4] J.L. Chaboche, Damage induced anisotropy: on the difficulties associated with the active/passive unilateral condition, International Journal of Damage Mechanics 1 (1992), 148-71.

[5] J.L. Chaboche, Development of continuum damage mechanics for elastic solids sustaining anisotropic and unilateral damage, International Journal of Damage Mechanics 2 (1993), 311-29.

[6] J.L. Chaboche and P.M. Lesne, A non-linear continuous fatigue damage model, Fatigue and Fracture of Engineering Materials and Structures 11 (1) (1988), 1-17.

[7] J.P. Cordebois and F. Sidoroff, Damage induced elastic anisotropy, in J.P. Boehler (ed.), Colloques Internationaux du CNRS No 295-Comportement mécanique des solides anisotropes, Martinus Nijhoff, Boston, 1983, pp 761-74.

[8] A.A. Griffith, The phenomena of rupture and flow in solids, Philosophical Transactions of the Royal Society 221 (1920), 163-98.

[9] L.M. Kachanov, Time of the rupture process under creep conditions, Izvestiia Akademia Nauk SSSR 8 (1958), 26-31 (in Russian).

[10] M. Kachanov, Effective elastic properties of cracked solids: critical review of some basic concepts, Applied Mechanics Review 45 (8) (1992), 304-35.

[11] D. Krajcinovic, Damage mechanics, North-Holland Series in Applied Mathematics and Mechanics 41, Elsevier, 1996.

[12] D. Krajcinovic and G.U. Fonseka, The continuous damage theory of brittle materials. Part 1: general theory, Journal of Applied Mechanics 48 (1981), 809-15.

[13] J. Lemaitre and J.L. Chaboche, Mécanique des materiaux solides, Dunod, Paris, 1985, [translated as Mechanics of solid materials, Cambridge University Press, 1994].

[14] A.B. Lennon, A stochastic model of damage accumulation in acrylic bone cement. Application to failure of cemented hip replacement, PhD thesis, University of Dublin, 2003.

[15] B.A.O. McCormack and P.J. Prendergast, Microdamage accumulation in the cement layer of hip replacements under flexural loading, Journal of Biomechanics 32 (1999), 467-75.

[16] S. Murakami, Mechanical modelling of material damage, Journal of Applied Mechanics 55 (1988), $280-6$. 
[17] B.P. Murphy, On damage accumulation of acrylic bone cement, PhD thesis, University of Dublin, 2001.

[18] B.P. Murphy and P.J. Prendergast, On the magnitude and variability of fatigue strength in acrylic bone cement, International Journal of Fatigue 22 (2000), 855-64.

[19] B.P. Murphy and P.J. Prendergast, The relationship between stress, porosity, and nonlinear damage accumulation in acrylic bone cement, Journal of Biomedical Materials Research 59 (4) (2002), $646-54$.

[20] M.H.J.W. Paas, P.J.G. Schreuers and J.D. Janssen, The application of continuum damage mechanics to fatigue failure mechanisms, in J.F. Dijksman and F.T.M. Nieuwstadt (eds), Integration of theory and applications in applied mechanics, Kluwer Academic Publishers, 1990, pp 49-63.

[21] P.C. Paris and F. Erdogan, A critical analysis of crack propagation laws, Journal of Basic Engineering, Transactions of the ASME 85 (1963), 528-34.

[22] R.H.J. Peerlings, R. de Borst, W.A.M. Brekelmans, and M.G.D. Geers, Localisation issues in local and nonlocal continuum approaches to fracture, European Journal of Mechanics A/Solids 21 (2002), $175-89$.

[23] K. Saanouni, J.L. Chaboche, and P.M. Lesne, On the creep crack-growth prediction by a non local damage formulation, European Journal of Mechanics, A/Solids 8 (6) (1989), 437-59.

[24] J.C. Simo and J.W. Ju, Strain- and stress-based continuum damage models-I. Formulation, International Journal of Solids and Structures 23 (1987), 821-40.

[25] J.J. Skrzypek, Material damage models for creep failure analysis and design of structures, in H. Altenbach and J.J. Skrzypek (eds), Creep and damage in materials and structures, International Centre for Mechanical Sciences (CISM) Courses and Lectures, 399, SpringerVerlag, Wien-New York, 1999, pp 97-166.

[26] J. Stolk, N. Verdonschot, B.P. Murphy, P.J. Prendergast and R. Huiskes, Finite element simulation of anisotropic damage accumulation and creep in acrylic bone cement, Engineering Fracture Mechanics 71 (2003), 513-28.

[27] S.P. Timoshenko, History of strength of materials, McGraw Hill, New York (republished: Dover Publications, Inc., NY, 1983), 1953.

[28] G.Z. Voyiadjis and T. Park, Anisotropic damage effect tensors for the symmetrization of the effective stress tensor, Journal of Applied Mechanics 64 (1997), 106-10.

[29] A. Wöhler, Über die festigkeitsversuche mit eisen und stahl, Zeitschrift für Bauwesen 20 (1870), 73 106, [English account of this work in Engineering, 11, 1871]. 